\title{
Double-frame silicon gyroscope packaged under low pressure by wafer bonding
}

\author{
Member Shinji Kobayashi, Kuniki Ohwada (Murata Mfg Co, Ltd) \\ Non-member Tetsuzo Hara, Takahiro Oguchi (Murata Mfg Co, Ldd) \\ Yasushi Asaji, Kaneo Yaji (Murata Mfg Co,Ldd)
}

\begin{abstract}
A new design for a "double-frame" vibratory silicon gyroscope is described. The gyroscope consists of two frames and corresponding springs. The inner frame has electrode fingers and is suspended from the outer frame, which has sensing springs. The outer frame is linked to the substrate by driving springs. This decoupling of the lateral driving and sensing motions makes the structure easy to design and improves the $\mathrm{S} / \mathrm{N}$ ratio. The gyroscope has a glass/silicon/glass structure with through-hole electrodes on one face. It is fabricated by the deep dry etching of silicon and low-pressure wafer-to-wafer anodic bonding. The noise equivalent angular rate is $0.8 \% \mathrm{~s}(\mathrm{rms}$, bandwidth: $40 \mathrm{~Hz}$ ), and the linearity is less than $1 \%$ for angular rates from -250 to $250 \%$. The overload resistance is over $1500 \mathrm{G}$ for $0.5 \mathrm{~ms}$, and the reliability of the package has been confirmed.
\end{abstract}

Keywords: gyrosoope, resonator, silioon, micromachining bonding

\section{Introduction}

Micromachined silicon gyroscopes will be useful in a variety of fields, such as automotive applications. The fact that they can be made very small provides such benefits as low cost and high reliability (high overload resistance, etc.).

Many designs for vibratory silicon gyroscopes have been proposed so far. Some employ the linear vibration of a mass along the substrate plane ${ }^{(1) \sim(3)}$, and others make use of out-of-plane movement ${ }^{(4),(5)}$. The basic layout of the resonator is closely related to the fabrication process, and each design has advantages with regard to the simplicity of the process or the superiority of the characteristics. From a practical point of view, the structure should be simple and able to be scaled down without much difficulty because of the inevitable demand for cost reduction. The use of only lateral motion can simplify the structure because driving and readout are easier than with out-of-plane motion. As for packaging, wafer-to-wafer bonding provides the advantages of high yield and low cost.

This paper describes a new type of bulk-micromachined vibratory silicon gyroscope fabricated by the deep dry etching of silicon and low-pressure wafer-to-wafer bonding. It uses only the lateral motion of the mass and is easy to design. The fabrication process is simple, and assembly is easy. So, it can be useful in a variety of fields.

\section{Basic design}

A schematic view of the new gyroscope is shown in Fig. 1. The basic design is almost the same as a previously reported one ${ }^{(1)}$ in that the driving and sensing directions are both parallel to the substrate. The gyroscope consists of two frames and corresponding springs. The outer frame is linked to the substrate by driving springs. The inner frame is suspended from the outer frame by sensing springs. When the outer frame is driven by an electrostatic force, rotation about the z-axis causes the inner frame to move in the $y$-direction due to the Coriolis force. So, both driving and sensing motions are in the $x-y$ plane. The separation of the driving and sensing springs decouples the driving and sensing modes of vibration to a great extent. The resonance frequencies of these two modes are $\omega_{d}=\sqrt{2 k_{d} /\left(m_{\text {out }}+m_{\text {in }}\right)}$, and $\omega_{s}=\sqrt{2 k_{s} / m_{\text {in }}}$, respectively. These frequencies are easy to set by adjusting the spring constants and the masses of the frames, and important parameters like $\left(\omega_{d}-\omega_{s}\right)$ are also easy to change. Each spring is designed to be pliable in one direction, and very stiff in the other direction. This separation is one of the major differences between this design and our previous one ${ }^{(1)}$. The resultant reduction in the mechanical coupling of the driving and sensing modes improves the angular rate resolution.

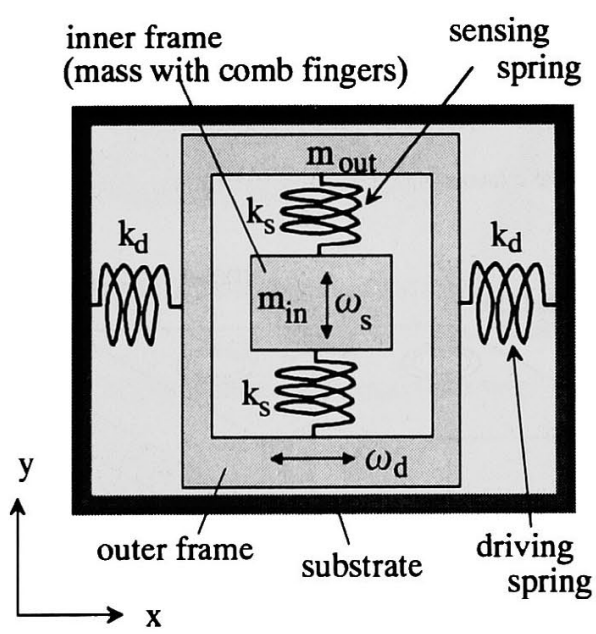

Fig.1 Schematic view of motion mode of doubleframe gyroscope. 
Figure 2 shows the basic layout of the device. The outer frame has comb fingers attached for electrostatic driving. The driving voltage has a sinusoidal wave form, and it causes driving vibrations along the $x$-axis. Under rotation, the inner frame is displaced along $y$-axis due to the Coriolis force. This displacement changes the capacitance between the movable electrode and the electrode fixed to the substrate. There is no need to make electrode patterns on the beams because the silicon is sufficiently doped to provide low conductivity. Hence, the fabrication process is quite simple, which helps reduce costs.

To obtain a high $\mathrm{S} / \mathrm{N}$ ratio, the resonator must have a high mechanical quality factor, $Q$, because sensitivity is proportional to driving amplitude, which in turn is proportional to $Q$. So, we employed low-pressure packaging ${ }^{(6),(7)}$ to reduce gas damping and to improve $Q$. Figure 3 schematically shows the packaged structure. The silicon resonator is sandwiched between two glass
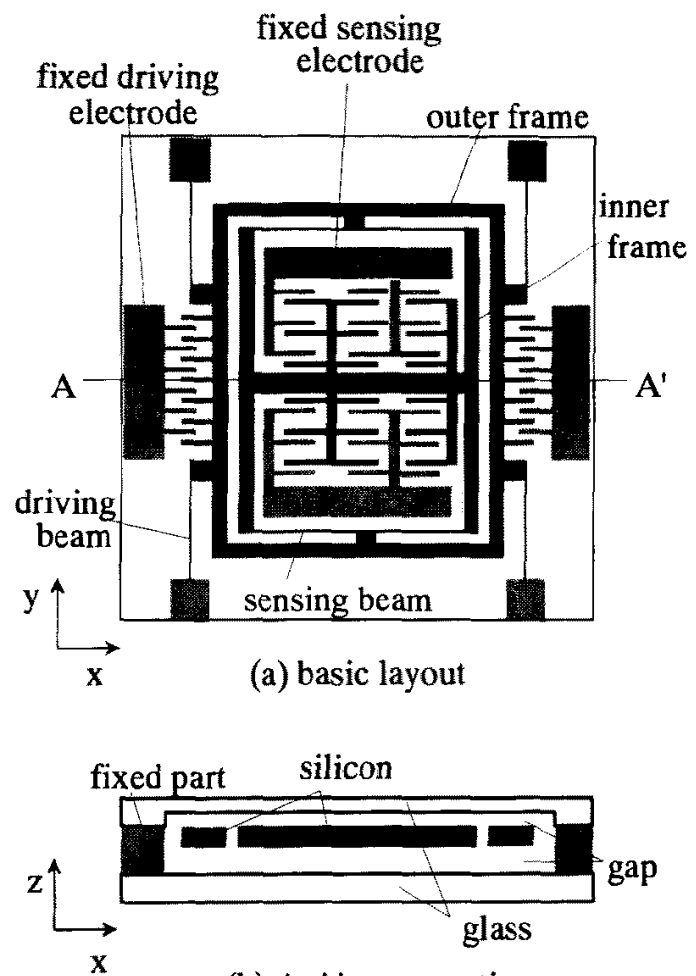

(b) $A-A^{\prime}$ cross section

Fig2 Basic layout of double-frame gyrosoope.

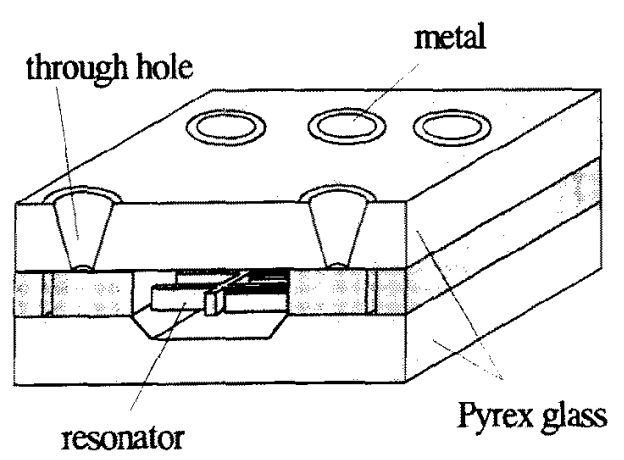

Fig 3 Schematic view of packaged structure. plates with the same thickness. This almost symmetrical design in the $x-y$ plane prevents serious effects from thermal stress and the levitation effect ${ }^{(5)}$; the latter is due to the unsymmetrical electrical field distribution which pulls the movable part upward or downward.

Since the device has a separate island, we use through holes in the glass to run electrodes to the detection circuit. The silicon resonator on the chip can be connected to the outer circuit in various ways, such as wire bonding, because the electrode pattern is formed on a glass plate, which is a very flat electrical insulator.

The sensitivity of the capacitance type sensor like the present one is very sensitive to the parasitic capacitance when the sensing capacitance is very small. Therefore, sensing capacitance well larger than parasitic capacitance is needed to provide a reasonable sensitivity for practical applications. It is easier to make a thicker resonator to increase the capacitance with bulk micromachining than with a surface micromachining process. So, this type of structure should enable considerable scaling down of $x-y$ area in the future.

\section{Fabrication process}

Several studies on the processing of glass/silicon/glass structures have been published ${ }^{(2)(8)}$. We employed low-pressure anodic bonding and the deep etching of silicon by RIE for the double-frame silicon gyroscope, as shown in Fig. 4.

a)

b)

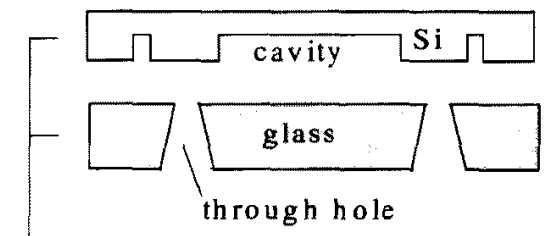

c)

d)

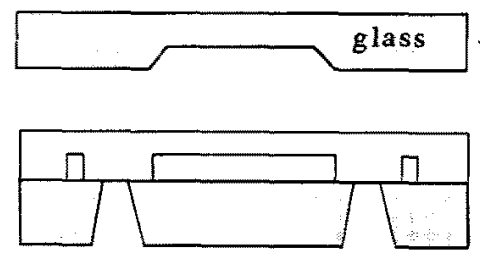

e)

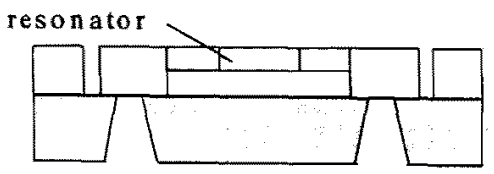

f)

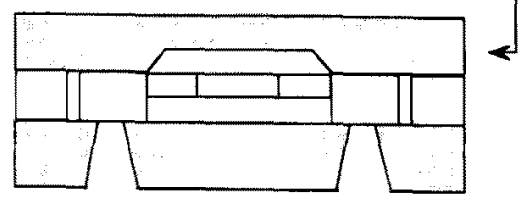

g)

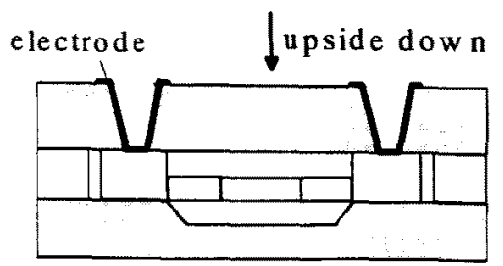

Fig-4 Fabrication process of packaged gyroscope. 
The silicon wafer is $200 \mu \mathrm{m}$ thick, and the glass plates are 400 $\mu \mathrm{m}$-thick Pyrex (Corning \#7740). The fabrication procedure is as follows: (a) The silicon wafer is etched by RIE to thin the movable part to about $30 \mu \mathrm{m}$. (b) Through holes are formed in one glass plate (through-hole glass) by sandblast. (c) A cavity is formed in the other glass plate (cover glass) so that the movement of the resonator is not restricted after bonding. (d) The throughhole glass is bonded to the silicon wafer by anodic bonding. (e) The comb fingers and beams are formed by RIE. The gap between the electrodes is about $2.5 \mu \mathrm{m}$. Figure 5 shows a SEM image of the etched structure. Figure 6 shows the part of fixed sensing electrodes and movable electrodes. (f) The cover glass is anodically bonded to the silicon wafer under a low pressure. The resultant pressure in the package was determined from the mechanical quality factor of the resonator and found to be in the range of 100 to $1000 \mathrm{~Pa}$. The anodic bonding of the silicon and glass hermetically seals the inner part with good reliability. (g) Metal layers $(\mathrm{Cr} / \mathrm{Au})$ are deposited on the through holes to provide electric contacts with an outer circuit. Finally, the wafer is diced into individual chips.

Figure 7 shows an optical photograph of a finished gyroscope chip, which is $5 \mathrm{~mm} \times 5 \mathrm{~mm} \times 1 \mathrm{~mm}$ in size. There are eight through holes: two for sensing ( $E_{1}$ and $E_{2}$ in Fig. 2), two for moving the frame (common potential), and the rest for driving (two holes for $D_{1}$ and other two holes for $D_{2}$ in Fig.2). Since the cavity is formed by wet etching in this case, the surface of the glass is smooth, and the resonator is visible through the glass.

\section{Characterization}

The characteristics of the gyroscope were measured using the readout circuit shown in Fig. 8. The gyroscope chip was attached to a printed circuit board with die-bonding adhesive. The electrical contact between the electrode on the chip and the pad on the board was made by wire bonding.

The small change in capacitance in the gyroscope was converted into a voltage change by the transistor in the $\mathrm{C}-\mathrm{V}$ converter. There are two basic signals: One corresponds to the capacitance between the inner frame and the fixed electrode $E_{1}$ (see Fig. 2), and the other corresponds to that for electrode $E_{2}$. The difference between the two basic signals reflects the amplitude of vibration along the $y$-axis (sensing mode), while their sum reflects that along the $x$-axis (driving mode). The driving mode must be kept stable to maintain the accuracy of angular rate detection, so a PLL (phase-locked loop) is used to drive the frames. AGC (Automatic Gain Control) controls the driving voltage in order to stabilize the vibration amplitude of the resonator.

The differential signal contains undesirable components, such as that produced by external linear acceleration, most of which are removed by a synchronous phase demodulator. The resonance frequency is about $5 \mathrm{kHz}$, so the effect of a typical acceleration (up to about $1 \mathrm{kHz}$ ) is easily reduced.

The reliability of the package was also evaluated ${ }^{(9)}$. Since the mechanical quality factor, $Q$, is very sensitive to the ambient pressure, the inner pressure was estimated from the measured $Q$ of the resonator or the outer frame of the device. The pressure dependence of resonance frequency and $Q$ were measured by putting the package on a actuator and vibrating it in a pressurecontrolled chamber. The inner pressure can be estimated from the

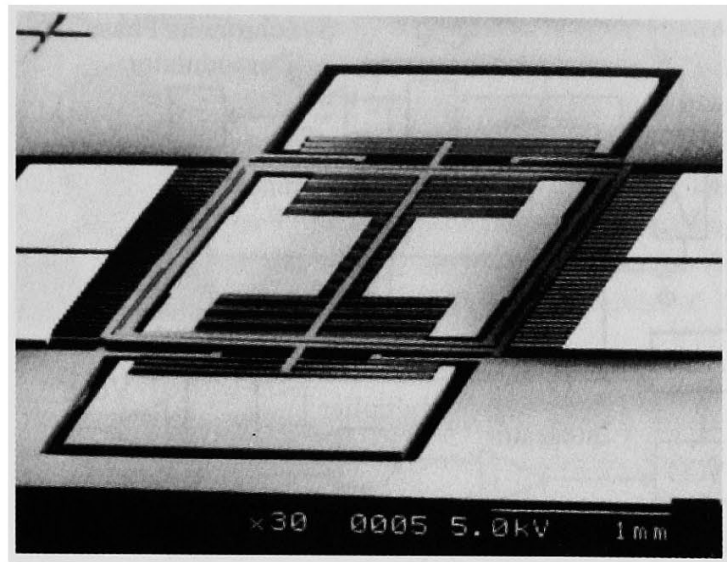

Fig5 SEM photo of device after the second etching of Si.

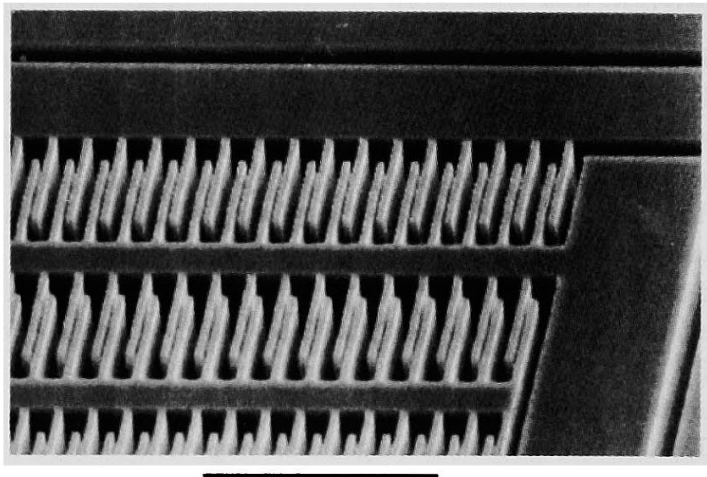

$100 \mathrm{~m}$

Fig.6 SEM photo of part of fixed sensing electrode and movable electrode.

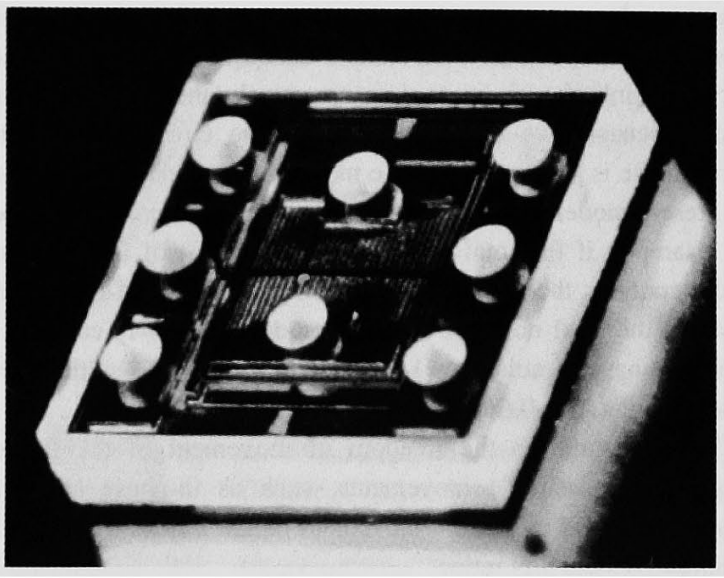

Fig.7 Optical photo of packaged structure.

measured $Q$ value.

The long-term stability was evaluated by leaving the device first in air, and then in a pressurized chamber under a pressure of $2 \times$ $10^{6} \mathrm{~Pa}$. A leak in the package would cause $Q$ to change about 20 times faster in the chamber than in air. Some environmental tests, such as a thermal shock test, were also performed to further assess the reliability of the package. 


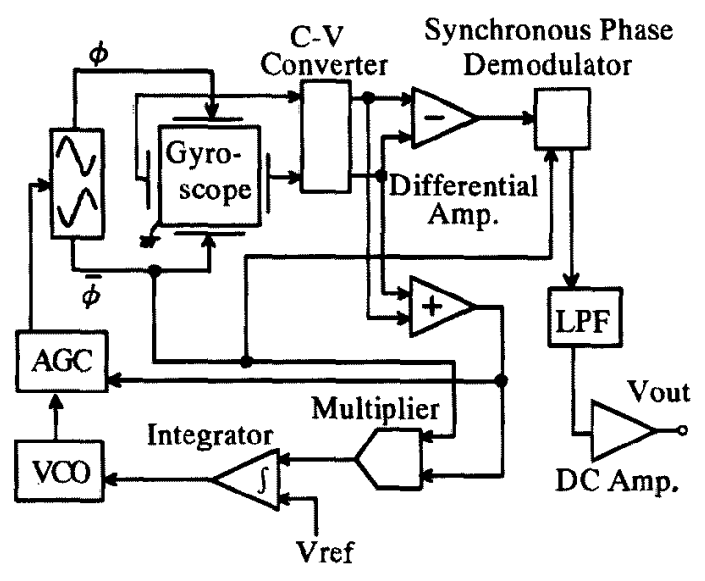

Fig.8 Block diagram of characterization circuit.

\section{Results and discussion}

Figure 9 shows an example of the relationship between output voltage and angular rate. The average sensitivity was set to 5.0 $\mathrm{mV} / \% / \mathrm{s}$, and the average linearity was less than $1 \%$ for angular rates from -250 to $250^{\circ} / \mathrm{s}$.

The measured characteristics are shown in Table 1. The noise equivalent angular rate is derived from voltage variation for $10 \mathrm{~s}$, and long-term drift is not included. This value is as low as $0.8 \%$ (rms) for a bandwidth of $40 \mathrm{~Hz}$. The overload resistance is over $1500 \mathrm{G}$ for $0.5 \mathrm{~ms}$, which is a typical load condition for automotive applications.

The decoupling of the driving and sensing vibration modes was found to greatly reduce the noise equivalent rate. The new model exhibited a noise which is about one third of the previous model ${ }^{(1)}$ in the same pressure range of around 100 to $200 \mathrm{~Pa}$. The noise contains the electric noise due to mechanical instability in the vibration of resonator, and the electric noise of the driving and sensing circuits. The ratio of the two components is not clarified. But we think the circuit noises are nearly the same for both models because we used almost the same circuits. When the circuit noise is the same for these models, the mechanical noise of the present model is not more than one third of the previous model. For example, if the total noise (amplitude $A=3$ ) of the previous model contains the circuit noise which is one fifth of total noise $(A=0.6)$, the total noise of the new model $(A=3 / 3=1)$ contains a small mechanical noise $(A=1-0.6=0.4)$ which is one sixth of the previous one $(A=3-0.6=2.4)$.

The restriction on the freedom of movement of the frames suppresses undesirable movements, such as in-phase elliptical movements. This makes the movement of the frames more stable and improves the $\mathrm{S} / \mathrm{N}$ ratio.

Even in the low-pressure package, the resonator exhibits a high overload resistance. This is a good characteristic for automotive applications, such as yaw rate sensing. It has often been pointed out that damping due to ambient gas is necessary to obtain a high overload resistance in a fine mechanical structure. But we think single-crystal silicon is durable because it is uniform and contains no inner or surface defects that might cause serious damage.

The long-term stability in a pressurized chamber $\left(2 \times 10^{6} \mathrm{~Pa}\right)$ is shown in Fig.10. There is no change in the inner pressure, even after $2577 \mathrm{~h}$. The leakage rate is accelerated by 20 times in the

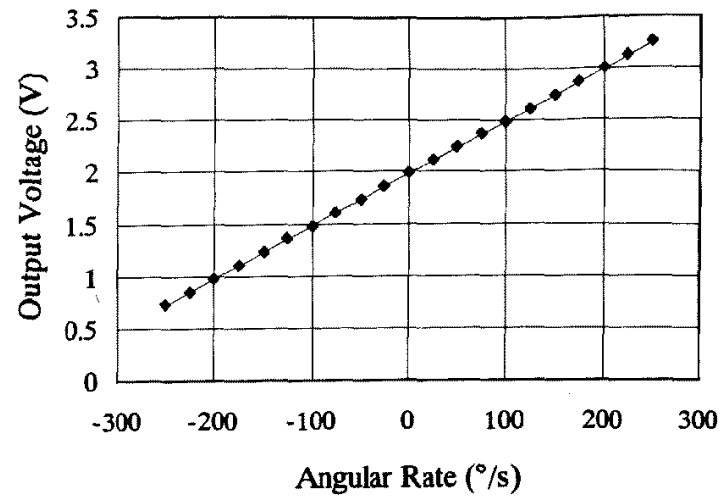

Fig. 9 Example of relationship between angular rate and output voltage.

Table.1 Characteristics of gyroscope.

\begin{tabular}{|l|c|l|}
\hline \multicolumn{1}{|c|}{ parameter } & value & \multicolumn{1}{c|}{ notes } \\
\hline sensitivity & $5.0 \mathrm{mV} / \mathrm{s}$ & $\begin{array}{l}\text { adjustable by changing } \\
\text { cirucuit gain }\end{array}$ \\
\hline lenearity & $<1.0 \%$ & \\
\hline effective range & -250 to $+250^{\circ} / \mathrm{s}$ & \\
\hline $\begin{array}{c}\text { noise equivalent } \\
\text { angular rate }\end{array}$ & $\begin{array}{c}0.8^{\circ} / \mathrm{s}(\mathrm{mms}) \\
\text { (bandwidth } 40 \mathrm{~Hz})\end{array}$ & measurement time: $10 \mathrm{~s}$. \\
\hline $\begin{array}{c}\text { over load resistance } \\
\text { operating temperature }\end{array}$ & $\begin{array}{c}-400 \mathrm{G}, 0.5 \mathrm{~ms} \\
\text { to }+85^{\circ} \mathrm{C}\end{array}$ & \\
\hline $\begin{array}{c}\text { duration of inner } \\
\text { pressure }\end{array}$ & $>5 y e a r s$ & $\begin{array}{l}\text { no change within } \\
\text { experimental error }\end{array}$ \\
\hline
\end{tabular}

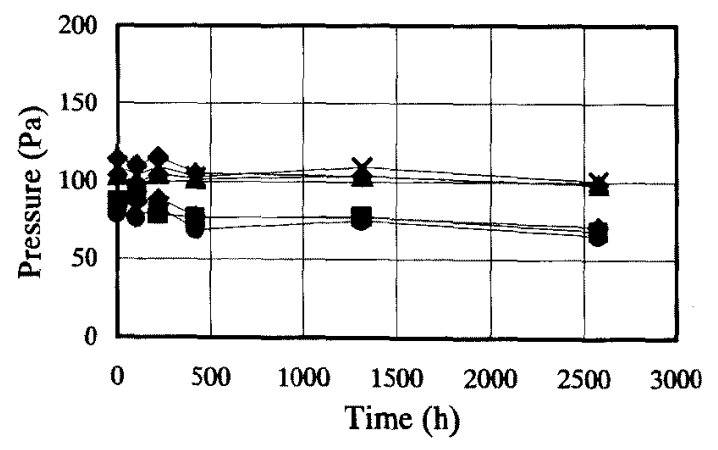

Fig.10 Stability of package under pressure of $2 \times 10^{6} \mathrm{~Pa}$.

pressured chamber, so the inner pressure can be maintained at least 5.9 years $(2577 \mathrm{~h} \times 20)$.

No leakage was observed after a thermal shock test of repeated cycles of lowering the temperature to $-45^{\circ} \mathrm{C}$ for $30 \mathrm{~min}$ and raising it to $85^{\circ} \mathrm{C}$ for $30 \mathrm{~min}$.

For these results of reliability test, anodic wafer-to-wafer bonding has been found to be applicable to the low-pressure packaging of a gyroscope. This small, hermetically sealed device is easy to attach at a variety of places on a circuit board or in an outer package, because there is little restriction on how the electrode pattern is drawn on the glass plate. The choice of 
assembly method is flexible, which helps reduce assembly costs.

\section{Conclusion}

A new design for a double-frame silicon gyroscope was described. The gyroscope consists of two frames and corresponding springs. The decoupling of the two linear motions improves the $\mathbf{S} / \mathrm{N}$ ratio, and the usage of only lateral motions can simplify the structure. The gyroscope was fabricated in a lowpressure package by wafer-to-wafer anodic bonding. The angular rate resolution $\left(0.8^{\circ} / \mathrm{s}(\mathrm{ms})\right.$ for bandwith $\left.40 \mathrm{~Hz}\right)$ and overload resistance $(1500 \mathrm{G}, 0.5 \mathrm{~ms})$ are good. The long-term stability of the package has been confirmed. So the device will be useful for automotive and other applications.

(Manuscript received Aug. 26, 1999, revised Nov. 1, 1999)

\section{References}

(1) H. Kawai, T. Shibano, K. Moriya, S. Kobayashi, and K. Ohwada, "Vibratory Rate Gyroscope with Lateral Movement for Driving and Sensing Using SOI Substrate", Technical Digest of the 16th Sensor Symposium, 1998, pp.37-40.

(2)M. S. Kranz, and G. K. Fedder, "Micromechanical Vibratory Rate Gyroscopes Fabricated in Conventional CMOS", Symposium Gyro Technology, Stuttgart, 1997, pp.3.0-3.8.

(3)S. S. Baek, Y. S. Oh, B. J. Ha, S. D. An, B. H. An, H Song, C. M. Song, "A Symmetrical Z-Axis Gyroscope with a High Aspect Ratio Using Simple and New Process", MEMS'99, 1999, pp.612-617.

(4)M. Lutz, W. Golderer, J. Gerstenmeire, J. Marek, B. Maihöfer, S. Mahler, H. Münzel and U. Bischof,, "A Precision Yaw Rate Sensor in Silicon Micromachining”, Transducers '97, 1997, pp.847-850.

(5)W. Geiger, B. Folkmer, U. Sobe, H. Sandmaier, and W.Lang, "New Designs of Micromachined Vibrating Rate Gyroscopes with Decoupled Oscillation Modes”, Transducers '97, 1997, pp.1129-1132.

(6)K. Funk, H. Emmerrich, A. Schilp, M. Offenberg, R. Neul, F. Lärmer, "A Surface Micromachined Silicon Gyroscope Using a Thick Polysilicon Layer”, MEMS'99, 1999, pp.57-60.

(7)M. Esashi, "Micromachining for Packaged Sensors", Transducers'93, 1993, pp.260-265.

(8)T. Sasayama, S. Suzuki, S. Tsuchitani, A. Koide, M. Suzuki, T. Nakazawa, and N. Ichikawa,"Highly Reliable Micro-machined Physical Sensors in Mass Production", Transducers '95, 1995, pp.687-690.

(9)T. Hara, S. Kobayashi, and K. Ohwada, "A New Fabrication Method for Low-Pressure Package with Glass-Silicon-Glass Structure and its Stability", Transducers '99, 1999, pp.13161319.
Shinj i Kobayashi (Member) received B.E., M.E. and Ph.D. degrees

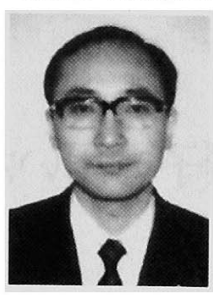
in applied physics from the University of Tokyo in 1978,1980 , and 1983, respectively. He is presently a manager of development section in Murata Mfg. Co., Ld. He has worked on the development of silioon micromachining technology and micromachined devices such as mechanical sensors. The Physical Society of Japan, The Japan Institute of Metals member.

Kuniki Ohwada (Member) received a B.S. degree in physics and a

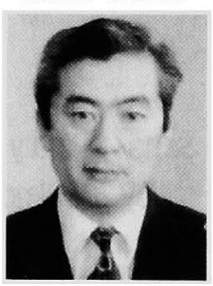

$\mathrm{Ph} . \mathrm{D}$. degree in electronic engineering from Tohoku University in 1967 and 1984, respectively. From 1967 to 1990, he worked at the electrical Communication Laboratories of Nippon Telegraph and Telephone Public Corporation, where he was engaged in research on thin film capacitors, CMOS devices and GaAs integrated circuits. He joined Murata Manufacturing Co. Ltd. in 1990, where he began R\&D on silicon micromachining. He is now a general manager of the development department for micromachining. He is a member of the institute of electronics, information and communication engineer, and the institute of electrical and electronics engineers.

Tet suzo Hara (Non-member) recieved a B.S. degree in applied physics

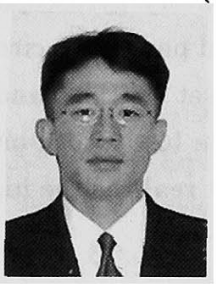
from Science University of Tokyo in 1990, and joined Research and Development Division of Murata Manufacturing Co., Ltd. in 1990. He has worked on microelectromechanical systems (MEMS) and development of acoelerometer and gyroscope. Japan Applied Physics Society member.

Takahi ro 0guchi (Non-member) received a M.E. degree in applied

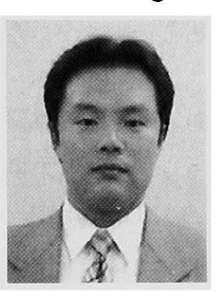
physics from Nagoya University in 1994 . He has worked at Murata Manufacturing Co, Ltd. sinœ 1994 in the field of silicon micromachining technologies.

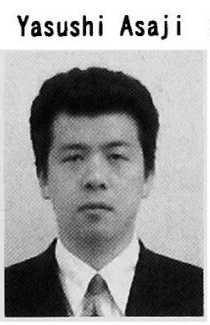

(Non-member) received a B.E. degree in chemistry from Tokyo Agriculture and Technology University in 1991, and joined Research and Development Division of Murata Manufacturing Co., Ltd in 1991. $\mathrm{He}$ has worked on development of Silicon Micromachining Technology and Surface Mount Technology.

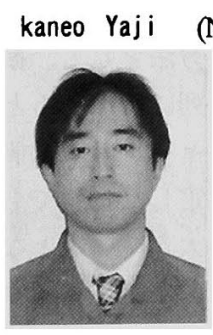

Non-member) received a B.E. degree in physics engineering from University of Electric Communication in1985. He worked at Iwatsu Electric,Co.,Ltd., from 1985 to 1992 . He has worked at Murata manufacturing $\mathrm{CO}$, Ltd since 1992, in the field of microsensors. 